\title{
INTRODUCTION
}

\section{Environnement numérique et musées}

\author{
CORINNE BAUJARD
}

Les musées du monde entier valorisent leur patrimoine culturel en proposant un accès élargi au contenu de leurs collections sur Internet. Du Guggenheim de Bilbao au MoMA (Museum of Modern Art) et au MET (Metropolitan Museum of Art) de New York, du Museum Lab de Tokyo à la Tate Gallery de Londres, plus de 600 institutions de 60 pays s'exposent désormais sur le web. Grâce à Google Art Project, mis en ligne dès 2011, on peut visiter par la technologie Street View tout ou partie des collections de 151 musées à travers 40 pays (32000 œuvres). Il est possible de parcourir virtuellement le Musée national de Toyko, de se promener parmi les sculptures aztèques du musée d'Anthropologie de Mexico ou s'attarder au palais des Offices de Florence.

Cette nouvelle relation à la culture bouleverse l'institution patrimoniale en charge de l'accueil du public, de la diffusion, de l'animation et de la médiation culturelle. L'environnement numérique repose sur une diversité de dispositifs médiatiques qui permet d'accéder à une connaissance culturelle, de rendre accessible le patrimoine universel.

En 2018, un plan national de numérisation de près de trois millions d'euros a été consacré aux projets de numérisation de contenus culturels. Le portail culture.fr met aujourd'hui à disposition gratuite un accès à plus de 7,5 millions de références : 5 millions d'images donnant accès direct à 73 bases de données. 
Ce sont 628 expositions sur 178 sites différents sur le patrimoine culturel français et étranger qui sont accessibles. Le projet Europeana offre également l'accès à 19 millions d'objets numérisés du contenu culturel et patrimonial et de la création contemporaine (archives, bibliothèques, musées, audiovisuel). Videomuseum, qui dispose depuis 2010 d'un réseau de collections publiques d'art moderne et contemporain à l'initiative de la Commission européenne, met en ligne 62 collections, 350000 œuvres de 35370 artistes. Le catalogue du patrimoine du ministère de la Culture comprend la numérisation des collections de 315 musées.

La base de données Google Arts \& Culture collabore avec plus de 1200 institutions dans le monde depuis 2016 et affiche sur son site plus de six millions d'œuvres numérisées, dont près d'un millier selon une technologie haute définition permettant d'accéder aux détails de la peinture invisibles pour le visiteur à l'œil nu. Les droits d'usage des images, des collections de fonds et des œuvres d'art en très haute résolution sont cédés par les musées à Google à titre gratuit qui finance la numérisation. Lorsqu'un tableau ne peut être prêté pour une exposition permanente, Google propose une projection de l'œuvre absente en haute définition comme la Naissance de Vénus de Botticelli à la galerie des Offices de Florence.

La numérisation du patrimoine occupe une place considérable au sein des pratiques culturelles et touristiques. Les études sur les comportements du public muséal interrogent les conditions d'accès aux collections publiques, la variété des parcours et la connaissance des œuvres. Une « googlecard» équipée d'une loupe en carton permet de visionner un petit film qui permet d'élargir le public. Chaque visiteur peut se constituer aujourd'hui sa propre exposition d'images répertoriées dans le monde entier. Le numérique constitue un changement pour les musées qui doivent tenir compte des nouvelles technologies pour communiquer avec le public, établir un contact plus actif permettant d'aller «hors les murs» et de construire le savoir d'une façon différente dans une perspective de diversité culturelle. C'est ainsi que la mission traditionnelle de conservation est remise en cause par de nouveaux dispositifs d'exposition, de parcours et d'accès aux collections.

Différents outils numériques réinventent déjà l'expérience de visite au musée: réalité virtuelle, réalité augmentée, applications mobiles, intelligence artificielle. Autant de technologies innovantes qui font apparaittre de nouvelles approches de valorisation des patrimoines, d'exposition, de médiation et d'expériences immersives et multi-sensorielles. Mais il convient désormais de mieux comprendre le rôle des tablettes tactiles, des applications Smartphone dans lesquelles le guide multimédia peut être téléchargé : des fiches sur les œuvres présentent différents contenus sur la biographie de l'artiste, des 
descriptions de tableaux. Au musée in situ, des bornes interactives permettent de s'orienter à l'aide d'informations pratiques, la billetterie est aussi en ligne. Les tables tactiles sont parfois dotées de casques, des sièges sont installés à proximité des œuvres avec des contenus accessibles à la loupe. Les visiteurs peuvent personnaliser leurs parcours, partager leur expérience sur les réseaux sociaux, découvrir grâce à la réalité augmentée les plus célèbres tableaux. La géolocalisation permet de recevoir des informations lorsque le visiteur est devant un tableau, des images de l'artiste en train de peindre, ou un commentaire sur sa vie.

\section{En quoi l'environnement numérique influence-t-il l'expérience de visite muséale?}

Dans un tel contexte, ce numéro double de la revue Les Cabiers du Numérique coordonné par Corinne Baujard (professeure des universités à Lille) a été réalisé avec la collaboration d'une équipe d'auteurs français, québécois, suisses et camerounais, spécialistes en information et communication, en sociologie et en sciences de l'éducation. Ils révèlent l'intérêt de comprendre comment se construit la médiation dans les espaces d'exposition, la manière dont les pratiques, les comportements, les usages des visiteurs sont bouleversés par l'environnement numérique, mais aussi comment l'institution muséale s'en saisit. Tout en intégrant de nouveaux dispositifs d'exposition, de parcours et d'accès aux collections dans un contexte in situ ou virtuel, les communications font état de retours d'expériences de visite, et construisent des connaissances en relation avec les savoirs thématiques des différents parcours d'éducation et d'accès aux savoirs.

Le premier article présente une étude sur la réception de l'exposition permanente du Mémorial de Caen à partir des lunettes équipées de caméras : «Eye-trakers et musée: présentation d'une étude de réception en espace muséal» (Stanilas Hommet). Il fait apparaître un état des usages et des connaissances propres à l'utilisation d'un tel équipement en espace muséal, mais également les principales données obtenues et les analyses qui en découlent. Le numérique au service de la compréhension de l'expérience de visite révèle que c'est l'usage de cet équipement en situation de mobilité qui apporte des connaissances lors de l'expérience de visite d'une exposition.

Le deuxième article intitulé «The Social and Sociotechnical Interaction of Visitors at a Digital Museum Exhibition : the Montreux Digigal Heritage Lab» (Taniana Smirnova, Dominique Vinck) analyse les interactions sociales et 
sociotechniques des visiteurs dans une exposition de musée numérique. De nouveaux types d'activités, de formes d'art et d'exposition apparaissent parallèlement aux dispositifs de virtualisation et de communication. L'enquête porte sur le projet « Montreux Jazz Heritage Lab II », un espace muséal qui n’a jamais été organisé selon les normes muséales classiques, tout en proposant une dynamique de changement à prolonger dans d'autres projets muséographiques.

Le troisième article s'interroge sur l'intérêt d'explorer les applications mobiles et autres e-albums à partir de grandes expositions: "L'évolution de l'environnement numérique des musées: Des CD-Roms aux applications muséales et autres e-albums » (Mathilde Miguet, Françoise Paquienseguy). Les auteurs montrent comment les e-albums s'inscrivent dans la continuité des CDRoms fondés sur des logiques transmédia, tout en s'appuyant sur la stratégie désormais centrale du storytelling mise en place dans les grandes expositions. Les institutions muséales offrent ainsi une nouvelle médiation culturelle.

Le quatrième article poursuit l'interrogation de la médiation culturelle: "Vers un patrimoine de médiations interpersonnelles ? Cas de la restitution en musée par une tablette de la présence d'un ancien résistant » (Gawin Geoffroy). L'auteur construit sa réflexion sur le rapport de présence que peut instaurer une tablette diffusant des enregistrements audiovisuels d'un témoin auprès des visiteurs. L'étude insiste sur un nouveau type de patrimoine par des médiations interpersonnelles.

Le cinquième article présente une étude des sites internet de musées d'art et de sciences: "L'éducation muséale en ligne: entre savoir réticulaire, coopération et standardisation» (Cédric Boudjema). L'auteur s'attache à montrer que les sites web des musées organisent les conditions de visites muséales relatives au savoir, mais aussi les stratégies de communications d'éducation muséale.

Le sixième article prend en considération les usages des TIC dans un musée au Cameroun: "Usages des TIC au musée national du Cameroun: entre balbutiements et pistes de développement» (Martial Sylvain Marie Abega Eloundou, Ousman Mahamat Abba). Au moment où se posent des questions sur la restitution de l'important patrimoine culturel africain domicilié en France, les auteurs s'intéressent à l'usage des TIC au musée national du Cameroun, dans un contexte africain d'insuffisances de ressources et plus particulièrement d'interrogations sur la conservation et la valorisation du patrimoine culturel muséal. Selon une démarche interactionniste et transactionnelle, la réflexion révèle des caractéristiques ethniques et linguistiques facilitatrices de la compréhension et de l'assimilation des connaissances culturelles médiatisées, l'identification et l'enracinement culturels des publics par des objets 
patrimoniaux médiatisés, des artefacts numériques révélateurs de l'altérité et du sentiment d'appartenance culturelle et nationale.

Le septième article aborde la mise en ligne d'une plateforme EducArt: «Enjeux techniques et pédagogiques associés à l'appropriation de la plateforme muséale numérique EuducArt par des enseignants du secondaire du Québec » (Marie-Claude Larouche, Katryne Ouellet, Denis Simard, Mélanie Deveault). Une recherche évaluative interroge les enjeux associés à l'appropriation de la plateforme numérique EducArt lorsque le Musée des Beaux Arts de Montréal ne fournit aucune autre forme de médiation aux enseignants du secondaire.

Le huitième article étudie le devenir du patrimoine numérique : «Musées et environnement numérique : quelles stratégies des professionnels des musées ?» (Emmanuelle Chevry Pebayle). Il insiste sur la prise en considération du numérique dans l'accès et la compréhension des savoirs, les possibilités de parcours de visite in situ ou en ligne.

Depuis une vingtaine d'années, de nouvelles modalités d'accès du public aux collections donnent une vision renouvelée des parcours de visite. Le visiteur voit son expérience muséale se transformer lors de la visite des collections. Il peut bénéficier de nouvelles modalités d'accès afin d'intégrer le contexte de son activité à l'exercice de sa démarche. La numérisation de l'environnement numérique occupe une place considérable au sein des pratiques culturelles et touristiques. Les musées du monde entier valorisent les espaces de visite. Les études sur les comportements du public muséal interrogent les conditions d'accès aux collections publiques. L'extension des espaces d'exposition multiplie la variété des parcours d'accès et de connaissance des œuvres. Il en résulte que la numérisation du patrimoine révèle des incertitudes scientifiques qui dépendent de la capacité à préserver la vocation muséale auprès du public des musées qui engage une réflexion sur lui-même dans son rapport à la culture, à une conception de la vie.

L'intérêt pour le rapatriement d'objets muséaux vers les pays d'origine constitue un enjeu de taille pour les institutions muséales concernées par la question des restitutions (rapport sur la restitution du patrimoine culturel africain, vers une nouvelle éthique relationnelle de Felwine Sarr et Bénédicte Savaoy, 23 novembre 2018). Ces mutations posent des questions éthiques aux responsables qui s'efforcent d'intégrer efficacement les nouvelles missions muséales. Si de nombreuses manifestations sont représentatives de l'ambition internationale, elles ne doivent pas servir de prétexte à une instrumentalisation du patrimoine éloignée du service public. C'est plus que jamais une responsabilité sociale que de conserver les biens collectifs pour les futures générations. 
L'environnement numérique peut être analysé à partir de ce qui s'observe dans l'exposition ou à partir ce qui s'y dit. Il faut prendre en compte les multiples situations à partir desquelles les expositions prennent sens, suivre des parcours de visite. D'emblée, il apparait que les interactions permettent de croiser intellectuellement d'autres visiteurs, venus au même moment, dans un même espace. C'est repenser la question du savoir et de l'éducation culturelle. Observer des visiteurs scolaires ou adultes qui regardent des tableaux, lisent des cartels ou des écrans, flânent, communiquent entre eux, permet déjà d'interpréter de l'expérience immédiate et sensible (Dewey, 1915, p. 46). L'éducation au musée revient à donner concrètement du sens à une dimension réflexive à laquelle invite la recherche sur les musées centrée sur l'action sociale du visiteur ou l'individualisation dans le collectif. Sur le plan pédagogique, John Dewey souhaite rapprocher l'art du quotidien en tant qu'expérience de la vie, car le savoir est une question de sensibilité qui peut nous servir. L'esthétique participe collectivement aux subjectivités multiples qui émergent dans l'interaction des systèmes humains. Il s'agit d'une mobilisation des savoirs appelant un usage «de soi par soi» qui engage une expérience, une mise à distance de soi et de mise au travail de sens. C'est progressivement que le visiteur élabore ses propres outils conceptuels cognitifs et intellectuels nécessaires à l'appréhension de son expérience muséale.

Que nous révèle l'environnement numérique sur la façon d'apprendre au musée ? Les modalités de déplacement du visiteur dans un environnement numérique peuvent-elles générer une valeur éducative, de nouvelles pédagogies ? L'expérience participante du chercheur dans les suivis de parcours favorise-t-elle les modalités de la connaissance interprétées par les applications muséales?

Visiter un musée permet de comprendre les relations sociales qui se nouent dans l'apprentissage, mais aussi le sens que l'on attribue à l'exposition, plutôt que la dimension pédagogique favorisant les interactions sociales. Afin de comprendre la démarche du visiteur individuel, il s'agit de saisir les valeurs, attitudes et préférences permettant de découvrir une collection. Tout contexte social a une influence sur le processus d'apprentissage qui impacte la réussite d'une exposition, ouvre des perspectives d'expérience sociale. Les musées évaluent l'expérience muséale en termes d'acquisition de connaissances et s'orientent vers les notions d'usage, d'appropriation et de création de sens lorsque le visiteur peut les rapprocher de sa propre expérience et qu'il apparaît aussi comme auteur de l'exposition. Le musée est un environnement social qui a un impact sur la pratique de visite, l'interaction sociale au niveau de l'expérience sociale, un espace où l'on vient pour se comprendre soi-même. 
En sciences de l'information et de la communication, en sciences de l'éducation, c'est un apprentissage qui a du sens, relève d'une expérience tant sur le plan social que physique, intellectuel et affectif. Le contexte social et physique de même que la motivation jouent un rôle certain au cours du processus d'apprentissage qui prend en compte l'acquisition de faits et de concepts. C'est également la mise en application des idées, des changements d'attitude, des expériences esthétiques, des interactions sociales, autant de facteurs qui contribuent à l'apprentissage. Le rôle que joue la motivation dans les choix effectués par le visiteur, et son insistance à acquérir des connaissances au musée, est un élément essentiel de l'apprentissage.

On assiste à la multiplication d'expositions qui deviennent des environnements scénographiques et impliquent le visiteur se réalisant dans l'espace d'exposition (lunettes, caméra). La présentation des œuvres varie selon la représentation qu'en possède le public. Le musée devient un espace social, une expérience de transmission sociale. La qualité des apprentissages dépend du contexte d'exposition car le visiteur mobilise souvent son attention au service de son propre intérêt. Or, les stratégies personnelles l'emportent généralement sur les stratégies de partage social. Les dispositifs sur lesquels s'appuie la transmission des connaissances ne doivent pas être négligés. Le regard académique se porte sur les questions émergentes en privilégiant la cohérence des usages sociaux dans les différents espaces. On note un double effet dès lors que les visiteurs sont acteurs, mais aussi producteurs de connaissances qui transforment les représentations des dispositifs. On retrouve le cadre théorique qui encourage la multiplication des fonctionnalités (supports, bases de données, sites personnels, blogs, environnements virtuels) pour échanger des contenus en ligne. Les usages sociaux modifient la stratégie muséale car de nouvelles compétences qui n'avaient pas été jugées indispensables jusqu'à présent apparaissent dans le processus de construction des réseaux de savoirs. Une véritable innovation se doit de prendre en considération cette réalité des usages et favoriser la réalisation des solutions déployées.

Le musée appartient à plusieurs champs sociaux et entretient des interactions avec l'environnement dans le cadre d'une démarche qui assure l'intéressement progressif des visiteurs des expositions selon les dispositifs proposés. On note différentes approches des missions des musées :

- L'interaction entre différents espaces d'exposition. Elle concerne les musées centrés sur le contexte d'exposition. Les représentations des visiteurs modifient l'environnement culturel reposant sur les dispositifs multimédias au sein du musée physique ou sur le site internet du musée, voire d'autres sites publics ou privés. Le musée repense ses frontières en tenant compte de toutes les démarches destinées à maintenir les savoirs existants pour construire des 
modalités de partage de connaissances. Le visiteur se dote d'une connaissance supplémentaire qu'il partage sur les sites sociaux. C'est au plan des possibilités d'interaction que le musée puise alors son changement institutionnel.

- La considération des visiteurs dans les musées centrés sur le contenu patrimonial fait que c'est la création de connaissances qui est privilégiée. On s'appuie sur des logiques collectives : plus les visiteurs sont intégrés dans les processus de connaissances des objets exposés, plus on obtient leur satisfaction. Dès lors que la concurrence dans la culture impose de se différencier sur des contenus, les enjeux esthétiques consistent à produire du sens afin de susciter la visite.

De l'interaction à la considération, la médiation numérique intègre la transmission des connaissances et l'accès aux contenus. Si les changements de signification sociale modifient l'attention vers les visiteurs au détriment des objets d'exposition, les dispositifs orientent la vocation du musée. La diffusion de contenus offre une grande liberté qui permet de construire ses propres connaissances scientifiques, d'en faire une action de construction culturelle. Dès lors qu'il existe une diversité de visites différentes et complémentaires, proche de l'objet d'exposition, le public ne limite pas son expérience au seul espace physique, il peut la prolonger dans la sphère privée. Dans les espaces, l'environnement est soumis à un principe dont il est issu, ce qui pose la question de la nature subjective des œuvres. On est confronté à un contexte de significations partagées qui rapproche le patrimoine d'une activité sociale.

Les travaux présentés dans le cadre de ce numéro témoignent souvent de problèmes concrets auxquels se heurte la mobilisation de champs théoriques dans un environnement institutionnel. Les comportements apparemment déterminés par des représentations et les processus sociaux demeurent complexes, surtout lorsque les visiteurs recherchent des connaissances adaptées à leur pratique de visite. Si l'espace muséal est un lieu d'interaction et de coordination entre acteurs, la structure est un lieu de mémoire où l'ajustement mutuel est envisagé toutes les fois qu'un schéma semble convenir pour être partagé dans le collectif.

Un dispositif de médiation permet de construire et de repérer un ensemble de compétences indispensables en assurant des compromis élaborés pour légitimer les dispositifs. La coordination facilite l'élaboration de référentiels d'interactions. Si des expositions ont pour effet d'encourager la visite réelle du musée, elles ne sont jamais exclusivement orientées vers une politique de diffusion des connaissances : elles jouent également le rôle d'un espace public. L'introduction de dispositifs interactifs permet de maitriser la situation, de faire des choix et de visualiser la complexité des interactions sociales avec le public. 
Il convient donc de caractériser la médiation soumise aux choix négociés collectivement dans un environnement social partagé. Les musées dépendent de connaissances patrimoniales de moins en moins précises, aux frontières du numérique et du culturel. Le processus d'innovation est d'une grande complexité puisqu'il revient à articuler les données techniques (programmes, logiciels, bornes interactives, tablettes, caméras), l'organisation de contenus de l'œuvre (scénarios, liens, écriture) et des usages sociaux (jeu, visite, création d'auteur).

Certains musées ont reconnu que les changements de signification sociale orientent l'attention vers les visiteurs au détriment des objets d'exposition dont les dispositifs bouleversent la vocation du musée. La relation au patrimoine doit permettre au visiteur de relier les espaces de connaissances, d'échange et de partage. Le système social muséal revêt des formes de communication tout en proposant des histoires de vie culturelle des objets exposés. Dès lors qu'il existe une diversité de visites différentes et complémentaires, les musées se trouvent dans une mission de service public qui reconfigure l'espace public en fonction des formes de l'expérience du visiteur. Le musée est un lieu de savoir et d'éducation, c'est à ce titre qu'il sert l'intérêt général et démocratique.

Les musées se pensent eux-mêmes comme ils pensent la société : en termes de rupture avec le passé et non de continuité. Si leur conception de l'exposition du patrimoine ne simplifie pas la recherche, le regard rétrospectif sur le public qui souhaite comprendre l'art, l'histoire et les techniques nécessaires pour conduire l'apprentissage. Environnement numérique et musées contribue à construire une pédagogie humaniste à la fois comme moyen d'acquisition de nouvelles connaissances culturelles et comme résultat d'une activité qui dépend des interactions des visiteurs avec l'environnement culturel au sein de différents univers. C'est une manière de se demander si l'universalité nait d'un espace tel qu'il se révèle par le biais de sa destination muséale ou de la mutation qui conduit le visiteur à transporter son propre univers. Autant dire que l'identité culturelle du musée, dont la vocation universelle reste très largement à définir, a beaucoup de difficultés encore à s'harmoniser avec la diversité de notre environnement numérique.

Un grand merci au Comité de lecture de ce numéro.

\section{Références bibliographiques}

Baujard C. (2018). Musées et management, ISTE Editions, Londres.

Baujard C. (2013). Du musée conservateur au musée virtuel, Hermès-Lavoisier, Paris.

Benghozi P-J (2011). L'économie de la culture à l'heure d'internet: le deuxième choc, Esprit juillet, p. 53. 
Bowen J., Bennett J. et Johnson J. (1998). Visiteurs virtuels et musées virtuels : Public, nouvelles technologies, musées, Publics et musées, $\mathrm{n}^{\circ} 13$, p. 109-127.

Clair J. (2007). Malaise dans les musées, Flammarion, Paris.

Conforti M. (2000). La tradition éducative et le concept des musées des beaux-arts aux Etats-Unis, Le regard instruit, J. Gallard, (coord.) la Documentation Française, Paris, p. 60.

Dewey J. (1915). L'art comme expérience, Folio Essais, rééd. 2014, Paris, p. 46.

DiMaggio P. (1987). Classification in Art, Audience Studies of the Performing Art and Museum, American Sociological Review, 52, p. 440-455.

Flichy P. (2012). Le sacre de l'amateur : sociologie des passions ordinaires à l'ère numérique, Seuil, Paris.

Flichy P. (1995). L’innovation technique, La Découverte, Paris.

Goodman N. (1984). The End of The Museum ?, L. Aagaard-Mogensen (ed.) The Ida of the Museum, Philosophical, Artistic and Political Questions, p. 27.

Greffe X. (2014). Les mises en scène du patrimoine culturel, Presses Universitaires du Québec, Canada.

Hoptman G.H. (1992). The Virtual Museum and Related Epistemological Concerns, Sociomedia : Multimedia, Hypermedia and the Social Construction of Knowledge, Ed. by Edward Barrett, Cambridge, Mass, MIT Press, p. 141-159.

Janes Robert R. (2009). Museums in a Troubled World : Renewal, Irrelevance or Collapse? Routledge éd., Abingdon, NY.

Kazerouni A. (2017). Le miroir des cheikhs. Musée et politique dans les principautés du golfe Persique, PUF, Paris.

Keene S. (1998). Digital Collections: Museums in the Information Age, ButterworthHeinemann, London.

Langley A., Smallman C., Tsoukas H. (2013). Process Studies of Change in Organization and Management : Unveiling Temporality, Activity and Flow, Academy of Management Journal, vol. 56, $\mathrm{n}^{\circ} 1$, p. 1-13.

Leedy P. D., Ormrod J. E. (2019). Pratical Research : Planning and Design, Pearson, 12e ed. New Jersey.

Lévy P., (1995), Qu'est-ce que le virtuel ?, Editions de la Découverte, Paris. p. 13-19.

Tobelem J. M. (2017). Le nouvel âge des musées. Les institutions culturelles au défi de la gestion, Armand Colin, 4 éd., Paris.

Vidal G. (2012). La sociologie des usages. Continuités et transformations, Lavoisier, Paris. 